\title{
LAS POLÍTICAS DE GÉNERO EN ESPAÑA: RETROCESOS Y RESISTENCIAS EN TIEMPOS DE AUSTERIDAD ${ }^{1}$
}

\section{Resumen}

Este trabajo realiza un análisis de los principales retrocesos en materia de políticas de igualdad en España. En primer lugar, expone los cambios acontecidos en aspectos centrales de esta área competencial, como los organismos, los presupuestos o la agenda política en materia de igualdad. En segundo lugar, repasa las transformaciones en políticas clave, como las relativas al Estado de Bienestar y su impacto sobre la situación de las mujeres. Finalmente, expone las resistencias del movimiento feminista y de mujeres ante estos evidentes retrocesos. Como conclusión, plantea que la crisis económica y la presencia de gobiernos conservadores en el poder constituyen factores explicativos para entender esta involución.

Palabras-clave: políticas de género, España, retrocesos, crisis económica.

\section{Abstract}

\section{Gender Policies in Spain: setbacks and resistance in times of austerity}

This article analyses the setbacks experienced by gender equality policies in Spain. First, changes in regard to key elements of this policy field, like the institutional mechanisms, the budgets or the equality policy agenda, are mapped. Second, transformations concerning key policies such as the ones related to the welfare state, and their impact on the situation of women, are reviewed. Finally, the article addresses the resistances of the feminist and the women's movements against these remarkable setbacks. To conclude, it is argued that both the economic crisis and the presence of conservative governments in power represent explanatory factors to understand this involution.

Keywords: gender quality policies, Spain, setbacks, economic crisis.

\section{Resumo} ridade

As políticas de género em Espanha: retrocessos e resistências em tempos de auste-

Este artigo analisa os principais retrocessos nas políticas para a igualdade em Espanha. Em primeiro lugar, expõe as mudanças que ocorreram em aspetos centrais desta área de competência, tais como os organismos, os orçamentos ou a agenda política em matéria de igualdade. Em segundo lugar, revê as mudanças nas políticas-chave, tais como o Estado Providência e seu impacto sobre a situação das mulheres. Por fim, discute os pontos fortes do movimento feminista e de mulheres, de resistência a esses retrocessos óbvios. Em conclusão, alega que a crise económica e a presença de governos conservadores no poder são fatores explicativos para a compreensão desta involução.

Palavras-chave: políticas de género, Espanha, retrocessos, crise económica.

1 Este texto ha sido realizado gracias a una Beca Postdoctoral financiada por la Xunta de Galicia. 


\section{Introducción}

Las políticas de género se habían caracterizado por su elevado nivel de consolidación en las últimas décadas, de manera que se habían desarrollado notablemente aspectos clave como la arquitectura institucional, el marco normativo o las estrategias para promover la igualdad (Squires, 2007). Sin embargo, el impacto de la crisis económico-financiera ha invitado a los estudios más recientes a replantearse la solidez de estos avances. Así, este fenómeno ha sido concebido como una coyuntura crítica que ha permitido poner en cuestión conquistas que parecían irreversibles, y desafiar los proyectos feministas a través de la vuelta a ideologías de género que se creían superadas (Dahlerup y Leyenaar, 2013; Rubery, 2014). En este sentido, existe un consenso general respecto al carácter generizado de la crisis económica tanto en lo relativo a sus causas como a sus consecuencias (Walby, 2009). Numerosos análisis han optado por centrar su atención en las segundas, recogiendo la literatura respecto a crisis precedentes, que ya habían mostrado su concepción como ventana de oportunidad para cuestionar los avances en materia de igualdad (Karamessini, 2014). En el contexto actual, se han podido corroborar sus perniciosos efectos a través del estudio de aspectos esenciales como los retrocesos en materia de políticas sectoriales de igualdad, la falta de perspectiva de género en las medidas anti-crisis o el cuestionamiento del Estado de Bienestar (Bettio et al., 2012; European's Women's Lobby, 2012; 2009; Karamessini y Rubery, 2014). Sin embargo, resulta todavía difícil capturar la incidencia de la crisis en toda su amplitud y complejidad. Por ello, trabajos recientes han optado por afinar la conceptualización de dichos retrocesos y proponer tipologías concretas que contribuyan a poner en evidencia aquellos que resultan menos obvios y más difíciles de identificar. El trabajo de Rubery ha sido especialmente útil a este respecto y ha propuesto diferenciar entre los cambios que implican resignificar las políticas existentes (conversion), los que se basan en un relajamiento en el cumplimiento de las mismas (drift), y finalmente, aquellas modificaciones que procuran directamente un desplazamiento relativo a la ideología de género dominante en favor de un marco más conservador (displacement) (Rubery, 2014).

El caso español resulta especialmente pertinente para explorar los retrocesos en materia de igualdad en sus diferentes manifestaciones. En primer lugar, se trata de un país que había sido testigo de una notable expansión y consolidación de las políticas de género tanto en el nivel estatal como en el autonómico (Alonso, 2015a). De este modo, dichas actuaciones habían dado lugar a un área competencial diferenciada que presentaba organismos, estrategias e instrumentos propios, así como una agenda política específica (Bustelo, 2014). Este nivel de desarrollo había permitido al estado español ocupar un lugar no desdeñable en los estudios comparados existentes, situando algunas de sus actuaciones como un referente internacional (EIGE, 2012), y ser considerado un país que evolucionaba hacia un régimen de género más avanzado (Walby, 2009, citada en Lom- 
bardo, 2013). En segundo lugar, España constituye uno de los países europeos que ha sido azotado en mayor medida por la crisis económica y que ha destacado por la aplicación de severas medidas de austeridad, especialmente tras la llegada de gobiernos conservadores. Por todo ello, constituye un valioso caso de estudio a través del cual resulta posible explorar hasta qué punto la crisis económica representa una ventana de oportunidad para cuestionar unas políticas de igualdad que se creían consolidadas; y también indagar en los diferentes tipos de retrocesos propuestos por Rubery, que arrojan luz sobre aquellas involuciones que resultan más difíciles de detectar.

Este artículo aborda ambos ejes de estudio a través del análisis de la literatura más reciente relativa al caso español. En concreto, realiza un repaso de los retrocesos identificados durante el periodo de crisis (2008-2015) respecto a tres ejes que resultan fundamentales para la promoción de la igualdad: 1) los elementos clave que definen las políticas de género como área sectorial - organismos, presupuestos e instrumentos en materia de igualdad -; 2) la agenda política relativa a la igualdad; 3) y el retraimiento del Estado de Bienestar en áreas cruciales para la situación de las mujeres. Además, propone explorar la reacción del movimiento feminista y de mujeres ante estos cambios y su potencial como agente que genere resistencias y salvaguarde los avances registrados en las últimas décadas.

\section{Las políticas para la igualdad en España: Tres décadas de avances}

La promoción de la igualdad en España comienza su andadura durante la transición política con los primeros gobiernos democráticos. El primer punto de inflexión se produce con la creación del Instituto de la Mujer en 1983, que se convirtió rápidamente en un actor clave para situar la igualdad en la agenda de gobierno y para reformar un marco legal claramente discriminatorio (Valiente, 2006). No obstante, el desarrollo del Estado autonómico determinó que las políticas de igualdad adoptaran desde el inicio un carácter multinivel y que las Comunidades Autónomas adquirieran un claro protagonismo en este ámbito (Alonso, 2015a; Bustelo, 2014). Los Estatutos de Autonomía aprobados a inicios de los años 80 ya las incluyeron entre las competencias del nivel sub-estatal. Ello derivó en la progresiva creación de instituciones específicas, en muchos casos replicando fielmente la estructura del Instituto de la Mujer estatal (Bustelo, 2004). De modo similar, los planes plurianuales de igualdad emergieron de manera inmediata como el instrumento privilegiado para desarrollar actuaciones en este ámbito, siguiendo de nuevo el ejemplo del ejecutivo central.

Sin embargo, a medida que el proceso de descentralización avanzaba y las Comunidades Autónomas adquirían nuevas competencias y mayores recursos, su papel en la promoción de la igualdad adquirió más relevancia (Bustelo y Ortbals, 2007). Mientras que el nivel estatal fue el claro inspirador e innovador en las actuaciones hasta la década de los 90, en la actualidad el liderazgo corresponde 
al nivel sub-estatal (Alonso, 2015a). Las Comunidades Autónomas han sido pioneras en la introducción de medidas clave que posteriormente se han visto generalizadas. Por ejemplo, la principal norma a nivel estatal, la Ley Orgánica 4/2007 para la Igualdad Efectiva entre Mujeres y Hombres, fue aprobada por el Congreso de los Diputados cuando seis leyes de igualdad autonómicas ya estaban en vigor. Este tipo de normas, existentes hoy en gran parte de las autonomías, han representado un salto cualitativo respecto a los planes de igualdad, asegurando un marco normativo amplio que garantizaba actuaciones en aspectos tan diversos como la paridad en la representación política, las medidas antidiscriminación, o la implementación del mainstreaming de género. Esta última estrategia ha contado en los últimos años con un destacado desarrollo, por el cual no sólo ha sido adoptada de manera generalizada en planes y normas, si no que se ha introducido una amplia variedad de instrumentos para ponerla en práctica que han dado lugar a cambios en los procesos y resultados de las políticas públicas (Alfama y Alonso, 2015; Alonso, 2015a). De nuevo, las autonomías han jugado un papel destacado de manera que territorios como Andalucía, el País Vasco o Cataluña han sido pioneros en el lanzamiento respectivamente de mecanismos como los presupuestos con perspectiva de género, la evaluación sistemática de las políticas de igualdad o los informes de impacto.

De manera similar, el concepto de igualdad incorporado por las políticas en la última década ha tendido a ser más transformador. Así, por ejemplo las actuaciones en materia de lucha contra la violencia de género han evolucionado hacia una concepción estructural de las desigualdades por la cual este fenómeno ha pasado a ser abordado desde una perspectiva integral e indisociable de la transformación de las relaciones de género (Bustelo y Lombardo, 2007). Ello indica la transición hacia una ideología de género más proclive a la promoción de la igualdad (Rubery, 2014).

Esta progresiva consolidación y evolución de las políticas, que no se vio excesivamente interrumpida en periodos de gobiernos conservadores como el de José María Aznar (Bustelo, 2014), ha determinado que España ocupe un lugar destacado, por ejemplo, en aquellos análisis comparados realizados a nivel europeo. Así, en aspectos como nivel de desarrollo del mainstreaming de género se ha situado entre los cuatro primeros países de la $\mathrm{UE}^{2}$, mientras que sus experiencias en materia de lucha contra la mutilación genital femenina han sido consideradas prácticas con potencial de ser transferidas a otros países (EIGE, 2012). Igualmente, se ha identificado su evolución general desde un régimen de género más conservador hacia otro donde la situación de las mujeres en la esfera pública y privada había mejorado notablemente (Walby, 2009, citada en Lombardo, 2013).

2 Véase el ránking en http://eige.europa.eu/about/gender-mainstreaming/spain (última visita 1803-2015). 


\section{Retrocesos en tiempos de austeridad}

3.1. El cuestionamiento de la política sectorial de igualdad: presupuestos, organismos e instrumentos

La tendencia hacia una ampliación y mejora de las actuaciones en materia de igualdad se ha visto sin duda interrumpida con el inicio del periodo de crisis. Por lo que se refiere a los elementos claves que permiten delimitar a las políticas de género como un área sectorial diferenciada, se han producido retrocesos significativos. Por una parte, los organismos de igualdad, que han sido el dinamizador clave de estas políticas en las últimas décadas (Valiente, 2006), han sufrido tres tipos diferentes de retrocesos. En primer lugar, se ha producido la eliminación de instituciones de larga trayectoria como el Servizo Galego de Igualdade o el Instituto de la Mujer de la Región de Murcia, y de otras de corta vida pero con especial peso simbólico, como fue el caso del Ministerio de Igualdad del gobierno central que existió entre los años 2008 y 2010. Igualmente, estructuras del ámbito local que resultan centrales para la atención de grupos como las mujeres que sufren violencia - centros de información, casas de acogida etc. - han sufrido recortes muy significativos (Beteta, 2013) ${ }^{3}$. La crisis económica y la necesidad de racionalizar el tamaño del sector público aparecen como razones para eliminar estas estructuras (Alonso, 2015a), aunque su peso en los presupuestos totales sea ínfimo (San José, 2014). Igualmente, la adopción de una estrategia transversal como el mainstreaming de género se ha utilizado para justificar la escasa utilidad de los organismos específicos, fenómeno que ya había sido detectado con anterioridad en ámbitos como las instituciones europeas (Stratigaki, 2005).

En otros ejemplos, los organismos han visto degradada su posición jerárquica en el organigrama de gobierno, perdiendo su adscripción a áreas con una mayor capacidad de influencia. Ese ha sido el caso del Instituto de la Mujer a nivel central que en 2011 deja de estar situado a nivel de Secretaría de Estado y pasa a depender de la Dirección General por la Igualdad de Oportunidades, una entidad de menor rango (Lombardo y León, 2014). Finalmente, se produce una fusión de algunos de los organismos de igualdad con otras áreas de gobierno que apuntan hacia una apuesta por políticas de perfil poco transformador. Ese ha sido el caso de autonomías como Navarra donde las competencias de familia han sido añadidas a la nomenclatura de la principal institución en la materia, derivando en el Instituto para la Familia e Igualdad.

Los retrocesos en materia de feminismo institucional se han visto agravados por los recortes referidos a los presupuestos. Así, las partidas presupuestarias dirigidas específicamente a la promoción de la igualdad, que son fundamentales

Como ejemplo, de acuerdo con el Informe Sombra publicado en 2014, en diciembre de 2011 se cerraron 9 de las 10 Oficinas de Atención a las Víctimas de delitos en las Islas Baleares, servicio que existía desde 1989, y que es esencial para las víctimas de violencia de género. 
para garantizar la efectividad de los organismos, se han visto significativamente disminuidas desde el inicio de la crisis económica en 2008. Con la excepción de Andalucía, todas las autonomías, así como el nivel central, han reducido los recursos dirigidos a esta área competencial (Paleo y Alonso, 2014). En gran parte de los casos lo han hecho además de manera notable. Así, por ejemplo para el periodo 2009-2014 el gobierno central ha disminuido el presupuesto en un 34,4\%. Resulta interesante destacar además que el análisis del periodo correspondiente también a la etapa de bonanza económica arroja interesantes conclusiones respecto a las variables que explican dichos retrocesos. De hecho, si bien la presencia de crisis económica muestra sin duda una relación estadísticamente significativa con los recortes presupuestarios realizados, la variable color del partido político en el gobierno resulta igualmente significativa. Se observa que cuando el ejecutivo está formado por un partido o una coalición de centro-derecha hay 4,15 veces más posibilidades de que se reduzcan los recursos dedicados a las políticas de género (Paleo y Alonso, 2014).

En términos de instrumentos clave como planes y leyes de igualdad, el balance tampoco es positivo. Con respecto a los primeros, se han producido retrasos notables en su aprobación en casos como el del gobierno central. Así, a pesar de que la ley estatal de igualdad prevé la aprobación periódica de un plan estratégico de igualdad, el gobierno central prescindió de este instrumento durante la mitad de la última legislatura - periodo 2011-2013 - (San José, 2014). Las leyes de igualdad presentan retrocesos similares. Por una parte, diversas normas en este ámbito vieron interrumpido su proceso de aprobación por la llegada de gobiernos conservadores (Alonso, 2015a). Ese es el caso de las leyes de igualdad propuestas en Cataluña, Islas Baleares o Cantabria en los años más recientes. También el de la ley contra la discriminación que fue paralizada por el ejecutivo del Partido Popular en su llegada al poder en 2011, incumpliendo el mandato europeo de aumentar la protección para otros ejes de desigualdad como la orientación sexual o el origen étnico (Alonso et al., 2012). Por otra parte, mecanismos esenciales como el informe periódico que evalúa la efectividad del principio de igualdad, han sido simplemente ignorados, a pesar de que la ley estatal de igualdad obliga a realizarlos de manera bianual para permitir el aprendizaje y mejora en su implementación (Alfama y Alonso, 2015).

\subsection{El giro conservador en la agenda politica: A vueltas con la maternidad}

Otro de los aspectos distintivos de las políticas de género es la agenda política en materia de igualdad, esto es, los ámbitos temáticos que se pretende priorizar, especialmente a través de la iniciativa y el poder disuasorio de los organismos de igualdad. En el caso español, esta había sufrido una clara ampliación a lo largo del tiempo de modo que a temas tradicionales como el empleo y la educa-

ción, se habían añadido otros como la paridad en los cargos de representación o 
la diversidad de las mujeres. Igualmente, el concepto de igualdad inherente a las políticas llevadas a cabo se evidenciaba más transformador y estructural, en especial desde la llegada del ejecutivo de Rodríguez Zapatero en 2004 (Bustelo y Lombardo, 2007).

Sin embargo, en los años más recientes ha tenido lugar una evidente involución a este respecto. En lo respectivo al gobierno central, se ha producido un claro énfasis en áreas de trabajo consolidadas, de modo que la igualdad de oportunidades en el empleo, la conciliación de la vida personal y familiar y la violencia de género pasan a ser prácticamente los únicos ámbitos de intervención. Paralelamente, se produce un giro copernicano en lo referido a las políticas relativas a la salud sexual y reproductiva de las mujeres, un área que había adquirido gran protagonismo en los últimos años. El gobierno socialista había aprobado en 2010 una norma que daba respuesta a algunas de las tradicionales demandas del movimiento feminista para garantizar el aborto libre y gratuito en las primeras semanas de embarazo ${ }^{4}$. Desde un inicio, el Partido Popular, en consonancia con la opinión de la jerarquía eclesiástica, mostró una absoluta oposición al cambio normativo propuesto, aduciendo la necesidad de proteger el derecho a la vida del concebido no nacido - o nasciturus. Tras su llegada al poder en 2011, el ejecutivo popular situó entre sus prioridades la aprobación de un nuevo marco legal que revertiera los avances conseguidos y lanzó en 2013 un Anteproyecto de Ley Orgánica para la Protección de la Vida del Concebido y de los Derechos de la Mujer Embarazada. Este determinaba que se había de establecer un sistema legal para la defensa del nasciturus como bien jurídicamente protegido. Para ello proponía autorizar la interrupción del embarazo solamente en unos supuestos muy determinados - violación o grave peligro para la salud física o psíquica de la madre y otorgar un papel central al personal médico en el proceso de autorización. A su vez, sugería reforzar los sistemas de información orientados a ofrecer alternativas a la práctica del aborto y a advertir de sus riesgos para la salud de la mujer.

Como veremos, la oposición a esta norma por parte del movimiento feminista, así como su bajo nivel de aceptación social, derivaron en su retirada en el año 2014. Sin embargo, los principios y valores que han inspirado dicha propuesta sí han entrado a formar parte del marco normativo a través de la acción de diversas Comunidades Autónomas (Paleo y Alonso, 2014). Así, desde el año 2008 se han aprobado en España hasta 7 normas relativas a la protección de la mujer embarazada (Castilla y León, Murcia, Galicia, Castilla La Mancha, Comunidad de Madrid, La Rioja, Islas Baleares), mientras que un nuevo texto se encuentra en proceso de aprobación en Extremadura. Con la excepción de la ley castellano-manchega, impulsada por un gobierno del PSOE, todas ellas van en coherencia con la propuesta realizada a nivel estatal y muestran explícitamente su voluntad de proteger el derecho a la vida desde la concepción. En este sentido,

4 Ley Orgánica 2/2010, de 3 de marzo, de salud sexual y reproductiva y de la interrupción voluntaria del embarazo. 
recogen un conjunto de medidas centradas en proveer a las mujeres gestantes de información y asesoramiento acerca de los recursos existentes para el apoyo a la maternidad, o en su caso acerca de aquellos previstos para los procesos de adopción y acogimiento. No contemplan por lo tanto la autonomía de las mujeres para decidir sobre la continuación del embarazo y dirigen todos los esfuerzos a la culminación de la gestación. Este marco normativo implica además una reorientación del gasto, de modo que programas que antes se dirigían a subvencionar las actividades de las organizaciones de mujeres, benefician ahora a entidades dedicadas a la defensa del derecho a la vida, tales como la Red Madre ${ }^{5}$.

Este enfoque no resulta sorprendente si se tiene en cuenta que estas normas han sido aprobadas por parlamentos autonómicos con mayorías del Partido Popular y que, por lo tanto, responden a su claro objetivo de limitar los derechos reproductivos de las mujeres, enfatizar de nuevo su rol como madres y cuidadoras, y fomentar el modelo tradicional de familia. De hecho, este mismo partido ha liderado otros retrocesos en aspectos tan variados como el uso de anticonceptivos y el acceso a la reproducción asistida para lesbianas y mujeres sin pareja (Platero, 2014) o la aprobación de planes de promoción de la familia que sitúan a las mujeres como responsables de las tareas reproductivas. Todo ello nos retrotrae a unas políticas que incorporan un concepto de igualdad que más que ser transformador y desafiante de los roles y estereotipos de género, perpetúa las desigualdades, representando una clara involución respecto a las actuaciones de años precedentes (Paleo y Alonso, 2014). De hecho, colisionan con el marco político y jurídico anterior que había apostado por introducir conceptos como la corresponsabilidad de hombres y mujeres en las tareas de cuidado o por ampliar los derechos reproductivos de éstas últimas promoviendo su plena autonomía.

\subsection{El Estado social y las políticas que 'importan' a las mujeres}

Los cambios experimentados en las políticas de género como área sectorial tienen también su reflejo en su concepción como área de naturaleza transversal. Tal como hemos apuntado con anterioridad, el mainstreaming de género constituye una estrategia de actuación central para las políticas de género en España desde hace casi dos décadas. Sin embargo, la crisis económica ha derivado en la puesta en marcha de profundas reformas y de amplios planes de actuación que no han incorporado en ningún caso la perspectiva de género. Así, líneas de acción clave que han marcado las políticas sociales y económicas españolas tales como los Programas Nacionales de Reforma marcados por la Unión Europea,

5 A modo de ejemplo, en el año 2012 el 26\% de los fondos destinados a la promoción de la igualdad dirigidos a organizaciones de la sociedad civil en Galicia fueron destinados a entidades centradas en el apoyo a las mujeres embarazadas, muchas de ellas de cariz religioso. 
han ignorado de manera sistemática su posible efecto diferencial sobre mujeres y hombres (Lombardo, 2013).

Estos programas de ajuste se centran esencialmente en la reducción del gasto público y la apuesta por un marco de actuación basado en la austeridad y en el desmantelamiento del Estado de Bienestar. Ello ha implicado disminuir drásticamente los recursos dirigidos a sectores centrales como los servicios sociales, la educación o la sanidad 6 , e incorporar medidas liberalizadoras en ámbitos clave como el mercado laboral (Beteta, 2013). Los estudios más recientes se han ocupado de destacar que este tipo de políticas están lejos de ser neutras desde la perspectiva de género, sino que más bien han representado un claro retroceso en la situación de las mujeres y en la lucha por la igualdad (González y Segales, 2014; Lombardo y León, 2014). Entre las medidas que han tenido un impacto negativo más notable podemos mencionar a modo de ejemplo aquellas que han sido destacadas por el último Informe Sombra publicado en España ${ }^{7}$ :

- EMPLEO: Ya en el inicio de la crisis, las medidas de estímulo aplicadas se centraron en paliar la pérdida de empleo en sectores altamente masculinizados como la industria y la construcción, siguiendo el ejemplo de otros países (Lescheke y Jepsen, 2014). Sin embargo, las políticas de disminución del gasto público se han dirigido esencialmente a aquellos sectores donde el porcentaje de mujeres es especialmente significativo - en torno a un $54 \%-$, como son la educación o la sanidad. Las sucesivas reformas de la regulación del mercado laboral muestran esta misma falta de perspectiva de género. Así, se han introducido medidas como la pérdida de relevancia de los convenios colectivos de sector - donde se incorporaban planes y medidas de igualdad -, la flexibilización de la jornada de trabajo por necesidades de la empresa, o la eliminación de bonificaciones para las madres que se reincorporan al trabajo tras el parto o la excedencia por cuidado de hijos, medidas todas ellas que perjudican de manera evidente a las mujeres.

- EDUCACIÓN: Los recortes en el sector educativo han afectado a actuaciones que resultan especialmente relevantes para facilitar la conciliación de la vida laboral y personal. Así, las reducciones presupuestarias han supuesto, por ejemplo, la paralización del Plan Educa3, que buscaba garantizar la escolarización de los niños/as de 0 a 3 años ${ }^{8}$, o la drástica reducción de las becas de comedor o de transporte escolar, que son espepromoción social, de un 21,3\% en el fomento del empleo, de un 21,9\% en educación o de un $7 \%$ en sanidad (Beteta, 2013).

7 Informe Sombra 2008-2013 sobre la aplicación en España de la Convención para la Eliminación de toda Forma de Discriminación contra las Mujeres, 2014. 
cialmente importantes para incentivar la plena incorporación de las mujeres al mercado laboral.

- DEPENDENCIA: La Ley 36/2006 de Promoción de la Autonomía Personal y Atención a las Personas que Sufren Dependencia nació para construir un cuarto pilar del Estado de Bienestar que diera soporte a las tareas de cuidado, las cuales eran mayoritariamente realizadas por las mujeres ${ }^{9}$. Dicha ley ha sufrido un claro proceso de desmantelamiento respecto de los avances conseguidos en el periodo anterior a la crisis. Así, la prestación económica para cuidados en el entorno familiar se ha visto reducida entre un $15 \%$ y un $30 \%$, se ha eliminado la cotización a la Seguridad Social de los/las cuidadores/as no profesionales, y se ha aplazado la incorporación al sistema de los dependientes moderados/as. Ello tiene un claro efecto sobre la situación de las mujeres que vuelven a carecer del apoyo del sector público en la realización de tareas de cuidado.

- SANIDAD: El derecho a la atención sanitaria universal y gratuita se ha visto significativamente recortado en los años más recientes. Grupos como las personas migrantes en situación irregular han perdido su acceso al sistema público de salud. Ello tiene un especial efecto sobre las mujeres, ya que, por ejemplo, muchos de los casos de violencia de género eran detectados por los servicios sanitarios de atención primaria, los cuales podían activar los protocolos de actuación en este ámbito. De manera similar, aspectos como el co-pago de los medicamentos afectan de manera desproporcionada a las mujeres mayores, que son las que con mayor frecuencia presentan patologías crónicas.

Todos estos ejemplos identificados por el Informe Sombra ponen de manifiesto la falta de perspectiva de género de las medidas implementadas desde el inicio de la crisis y su pernicioso efecto sobre la igualdad. En este sentido, los datos indican que se han replicado fenómenos identificados en crisis anteriores tales como la provisión por parte de las mujeres de aquellos bienes y servicios como el cuidado, que debido a los recortes presupuestarios ya no son suministrados por el Estado - también denominado proceso de re-familiarización -, o el retroceso de los avances en igualdad conseguidos en épocas de bonanza económica (Gálvez y Rodríguez, 2011). Igualmente, se replican las tendencias detectadas en otros países, que también apuntan a la disminución del gasto público en materia de cuidado, y que han abierto el debate respecto a la posible presencia de involuciones respecto al régimen de género predominante (Leschke y Jepsen, 2014; Walby, 2013).

9 La feminización de los cuidados resulta más significativa en España que en la media de la UE, siendo uno de los países con mayor proporción de mujeres fuera del mercado laboral por cuidado familiar. 


\subsection{El movimiento feminista y de mujeres: ¿hay futuro para las políticas de igualdad?}

La sociedad española no ha permanecido ajena a los profundos cambios acaecidos en el marco de la era de la austeridad. El surgimiento del Movimiento 15M - también conocido como los/as Indignados - en el año 2011 fue sin duda el primer signo de la presencia de un cuestionamiento de los cambios políticos y económicos experimentados en el país, y un claro desafío a las posiciones dominantes enmarcadas en la máxima thatcheriana de no hay alternativa - there is no alternative -. Lejos de permanecer como un fenómeno aislado que finaliza cuando se desocupan las plazas, el 15M ha mostrado ser la semilla que ha dado lugar a una multiplicidad de movimientos sectoriales que han intensificado la lucha contra el denominado austericidio. Es sin duda necesario mencionar aquí las diferentes Mareas que nacieron para paralizar el desmantelamiento de los ámbitos elementales del Estado de Bienestar español, como son la sanidad - marea blanca -, la educación - marea verde - o los servicios sociales - marea naranja -; la Plataforma de Afectados por la Hipoteca (PAH), que ha liderado las reivindicaciones en materia de derechos habitacionales y paliado los devastadores efectos de los miles de desahucios de primera vivienda que se producen al año en España; o las Marchas de la Dignidad que agrupan a activistas de una variedad de organizaciones en su reivindicación de políticas que atajen la situación de emergencia social. Además, el sistema de partidos ha sufrido los efectos del mayor nivel de implicación de la sociedad civil, dando lugar a nuevas organizaciones donde la democracia directa constituye un eje central - como es el caso de Podemos - o a plataformas ciudadanas que concurren a las elecciones para competir con los partidos políticos tradicionales, identificados ahora con la vieja política.

El movimiento feminista no ha permanecido ajeno a este renacimiento de la sociedad civil y ha experimentado igualmente un incremento en su actividad e incidencia política en los años más recientes (García, 2014, 2015). Por una parte, los diferentes movimientos sociales han contado con un claro activismo de las mujeres, así como con la presencia de reivindicaciones específicamente vinculadas a la igualdad. Así, el 15M contó con comisiones feministas especialmente activas en diversas ciudades (Cruells y Ruiz, 2014), la marea violeta - formada por organizaciones feministas y de mujeres - ha incorporado las demandas en materia de igualdad en las protestas multicolor en defensa del Estado de Bienestar, y la PAH se distingue por el destacado liderazgo y participación de las mujeres.

Por otra parte, resulta innegable que el movimiento feminista español ha experimentado un resurgimiento al calor de uno de los mayores ataques ejercidos a los derechos de las mujeres españolas en décadas, el Anteproyecto de Ley Orgánica para la Protección de la Vida del Concebido y de los Derechos de la Mujer Embarazada. La respuesta fue de absoluta oposición y desencadenó uno de los picos de movilización más notables en toda su historia, así como la recuperación de las actuaciones a nivel estatal, que no habían resultado comunes en mucho tiempo dado el elevado nivel de regionalización del movimiento. En este sentido, 
ya en el año 2013, cuando el borrador de ley estaba siendo elaborado, se produjeron las primeras jornadas de lucha en todo el estado, con la realización por ejemplo de escraches en las sedes del Partido Popular (García, 2014). Igualmente, se comenzaron a gestar estructuras de coordinación que facilitaran actuaciones conjuntas, tales como la Plataforma Estatal Derecho a Decidir, que ha llegado a agrupar hasta 400 organizaciones de diferente índole - organizaciones de mujeres, sindicatos, partidos etc. -, y se reactivaron otras como la Coordinadora Estatal de Organizaciones Feministas, que contribuyó a organizar jornadas de lucha que abarcaron todas las autonomías (García, 2015). La acción coordinada de mayor visibilidad fue sin duda el denominado 'Yo Decido. Tren de la Libertad'. Una campaña que se inició en una pequeña localidad de Asturias y que llegó a congregar en Madrid a miles de mujeres de toda España el 1 de febrero de 2014, reivindicando el derecho a decidir libremente sobre su maternidad. Este acto contribuyó a dar todavía más notoriedad a las movilizaciones en el contexto internacional, actuando como complemento de campañas como 'No more hungers' dirigida a los miembros del Parlamento Europeo o la Red Federica Montseny, que comenzó a organizarse en 2013 en diversas ciudades europeas para ayudar a aquellas mujeres que tuvieran que desplazarse al extranjero para acceder a un aborto seguro.

Con todo ello, el movimiento feminista y de mujeres alcanzó un nivel de movilización extraordinario, que no se había producido en décadas. También consiguió incrementar notablemente el nivel de implicación de las mujeres jóvenes en la lucha por la igualdad, ya que por primera vez vieron seriamente amenazadas las conquistas realizadas por las generaciones precedentes (Alonso, 2015b). La activación del movimiento se vio además acompañada de una opinión pública especialmente contraria a la ley propuesta. Así, solamente un $10 \%$ de población afirmaba apoyar el cambio normativo, mientras que hasta un $60 \%$ era partidario de mantener la ley socialista basada en el establecimiento de plazos legales en los que interrumpir el embarazo ${ }^{10}$. Incluso entre electorado del Partido Popular el nivel de aprobación de la norma vigente alcanzaba un 35\%. Todo ello determinó que el gobierno retirara el anteproyecto de ley y que el propio ministro que lo impulsó abandonara su cargo, otorgando al movimiento feminista una celebrada victoria (García, 2015). Sin embargo, la voluntad del gobierno de perseverar en su agenda en materia de protección del derecho a la vida ha determinado que mantengan su intención de restringir el acceso al aborto de las menores de 18 años, así como todo el marco normativo en materia de protección de la mujer embarazada. Con todo ello se matiza el potencial del movimiento para revertir los amplios y diversos retrocesos experimentados y la necesidad de contar con otros actores aliados. 


\section{Conclusiones}

La crisis económica constituye sin duda un desafío de primera magnitud para la consecución de la igualdad de género. El análisis de la literatura relativa al caso español ha permitido, en primer término, explorar un ilustrativo ejemplo de cómo este fenómeno ha sido utilizado como una ventana de oportunidad para cuestionar las políticas de igualdad. Así, aunque este constituía un ámbito de actuación que se encontraba ampliamente consolidado, el periodo de recesión ha sido utilizado por los diferentes gobiernos, y particularmente por los de cariz conservador, para frenar su expansión y justificar retrocesos en aspectos elementales como los organismos de igualdad, la agenda política o los pilares que sostienen el Estado de Bienestar; todas ellas conquistas que parecían irreversibles. Con ello se refuerza la hipótesis de la literatura que identifica la crisis económica como coyuntura crítica y que señala su gran potencial para generar una notable involución en la situación de las mujeres.

En segundo lugar, el análisis de los trabajos realizados hasta la fecha permiten afinar en la identificación de diversos tipos de retrocesos, ejercicio que resulta fundamental para valorar su alcance real. Así, estos varían desde la conversión de las políticas existentes hasta un claro cambio de la ideología de género (Rubery, 2014). Resulta posible, en primer lugar, identificar procesos de resignificación que derivan en un cambio de contenido de las actuaciones en marcha (conversion). Ese es el caso, por ejemplo, de la implementación del mainstreaming, que se ha utilizado como coartada para eliminar políticas y organismos específicamente dirigidos a promover la igualdad. Ello a pesar de que se trata de una estrategia que, para tener un impacto real, necesita de la ampliación de la arquitectura institucional y en ningún caso su desaparición. En segundo término, el caso español ofrece también ejemplos de relajamiento en el cumplimiento de las políticas existentes (drift). La disminución de presupuestos, la falta de evaluación de los planes o la práctica paralización de actuaciones en vigor, como la ley de dependencia o el plan para la escolarización de niños/as de 0 a 3 años, ilustran este tipo de tendencias. Así, las medidas no son formalmente eliminadas pero carecen de efectividad en la práctica, volviéndose esencialmente retóricas. Finalmente, las políticas de igualdad en España ilustran de manera clara la presencia de procesos de desplazamiento o de cambio en la ideología de género dominante a favor de un marco conservador (displacement). Las nuevas regulaciones en materia de salud sexual y reproductiva, los planes de familia o las nuevas nomenclaturas de los organismos de igualdad muestran, de hecho, el giro copernicano en lo relativo a este ámbito. Las políticas de igualdad vinculan de nuevo a las mujeres con su rol como madres y cuidadoras, a la vez que cuestionan de modo claro su autonomía para decidir sobre su propio cuerpo, que se ve subordinada respecto al derecho a la vida del concebido no nacido.

El movimiento feminista y de mujeres no ha podido si no reaccionar para hacer frente a esta ofensiva. También la respuesta de la opinión pública y los movimientos sociales ha mostrado una clara oposición a reformas como las refe- 
ridas al desmantelamiento del Estado de Bienestar. Sin embargo, la amplitud y variedad de los cambios introducidos hace presagiar tiempos difíciles para las políticas de igualdad en España. Así, su continuidad no garantiza ni que éstas sean aplicadas en la práctica ni que su contenido sea dirigido a promover de manera efectiva la transformación de las relaciones de género. Es por lo tanto necesario realizar análisis que continúen profundizando en los diferentes retrocesos experimentados, especialmente en aquellos más sutiles y difíciles de percibir. Con ello será posible comenzar a construir una fotografía general acerca de los efectos de la crisis económica sobre la igualdad entre mujeres y hombres. Igualmente, se podrán percibir en mayor medida los cambios experimentados por el régimen de género a medio plazo, así como la utilización de este periodo como oportunidad para cambiar el marco dominante hacia otro de cariz más conservador.

\section{Referencias}

Alfama, Eva; Alonso, Alba (2015), «Las políticas de género en la Administración Pública. Una Introducción», Revista Vasca de Gestión de Personas y Organizaciones Públicas 8, 24-41.

Alonso, Alba (2015a), El mainstreaming de género en España, Valencia, Tirant lo Blanch.

Alonso, Alba (2015b), «Is Sisterhood under Threat? The Women's Movement and the Independence Referendum in Scotland and Catalonia", 4th European Conference on Politics and Gender 11-13 junio, Uppsala.

Alonso, Alba et al. (2012), «Institutionalizing intersectionality in Southern Europe: Italy, Spain and Portugal», in Andrea Krizsan; Hege Skjeie; Judith Squires (eds.), Institutionalizing Intersectionality?: The Changing Nature of European Equality Regimes, Bakingstone, Palgrave Macmillan, 148-178.

Beteta, Yolanda (2013), «La feminización de la crisis financiera global. La regresión del Estado de Bienestar en España y su impacto en las políticas de igualdad en España y de erradicación de la violencia contra las mujeres», Asparkía 24, 36-52.

Bettio, Francesca et al. (2013), The Impact of the Economic Crisis on the Situation of Women and Men and on Gender Equality Polices, Luxemburgo, Comissão Europeia.

Bustelo, María (2014), «Three decades of state feminism and gender equality policies in multi-governed Spain», Sex Roles, vol. 70, 9/10, (DOI 10.1007/s11199-014-0381-9), [em linha] disponível em http://link.springer.com/article/10.1007\%2Fs11199-014-0381-9 [consultado em 15/06/2014].

Bustelo, María (2004), La evaluación de las políticas de igualdad de género, Madrid, Catarata.

Bustelo, María; Ortbals, Candice (2007), «The Evolution of Spanish State Feminism: a Fragmented Landscape», in Joyce Outshroorn; Johanna Kantola (eds.), Changing State Feminism, Basingstoke, Palgrave Macmillan, 201-223.

Bustelo, Maria; Lombardo, Emanuela (eds.) (2007), Políticas de igualdad en España y en Europa, Madrid, Cátedra.

Cruells, Marta; Ruiz, Sonia (2014), «Political Intersectionality within the Indignados Social Movement», Intersectionality and Social Change 35, 3-25.

Dahlerup, Drude; Leyenaar, Monique (eds.) (2013), Breaking Male Dominance in Old Democracies, Oxford, Oxford University Press. 
EIGE (2012), Study to map the current situation and trends of FGM in EU27 Member States and Croatia. Country Reports, Vilnius, Instituto Europeu para a Igualdade de Género.

European Women's Lobby (2012), The Price of Austerity: The Impact on Women's Rights and Gender Equality in Europe, Bruxelas, EWL.

European Women's Lobby (2009), Women and the Economic Crisis - An opportunity to assert another vision of the world?, Bruxelas, EWL.

Gálvez, Lina; Rodríguez, Paula (2011), «La desigualdad de género en las crisis económicas», Revista de Investigaciones Feministas 2, 113-132.

García, Joana (2015), «Feminismos en tiempos de resistencias a la austeridad y confluencias por la ruptura con el 78», Anuario Movimientos Sociales 2014, Fundación Betiko.

García, Joana (2014), «Feminismos poniendo el cuerpo frente a la doctrina del shock y la misoginia institucional», Anuario Movimientos Sociales 2013, Fundación Betiko.

González, Elvira; Segales, Marcelo (2014), «Women, gender equality and the economic crisis in Spain», in Maria Karamessini; Jill Rubery (eds.), Women and Austerity. The economic crisis and the future for gender equality, Londres, Routledge, 228-247.

Karamessini, Maria (2014), «Introduction - women's vulnerability to recession and austerity: a different crisis, a different context», in Maria Karamessini; Jill Rubery (eds.), Women and Austerity. The Economic Crisis and the Future of Gender Equality, London, Routledge, 3-16.

Karamessini, Maria; Rubery, Jill (eds.) (2014), Women and Austerity. The Economic Crisis and the Future of Gender Equality, Londres, Routledge.

Leschke, Janine; Jepsen, Maria (2014), «Is the Economic Crisis Challenging the Prevailing Gender Regime? A Comparison of Denmark, Germany, Slovakia and the United Kingdom», Social Politics 21(4), 485-508.

Lombardo, Emanuela (2013), «Gender mainstreaming and policy responses to the economic crisis: the 'unintended consequences' of EU and national policymaking on Spanish gender equality policies», Congreso de la Asociación Española de Ciencia Política, Sevilla, 17-19 septiembre.

Lombardo, Emanuela; León, Margarita (2014), «Políticas de igualdad de género y sociales en España: origen, desarrollo y desmantelamiento en un contexto de crisis económica», Revista de Investigaciones Feministas 5, 13-35.

Paleo, Natalia; Alonso, Alba (2014), «¿Es solo una cuestión de austeridad? Crisis y políticas de género en España», Revista de Investigaciones Feministas 5, 36-68.

Platero, Raquel (Lucas) (2014), «Las políticas neoliberales contra los derechos sexuales de la ciudadanía», Anuario Movimientos Sociales 2013, Fundación Betiko.

Rubery, Jill (2014), «From 'women and recession' to 'women and austerity': a framework for analysis» in Maria Karamessini; Jill Rubery (eds.), Women and Austerity. The Economic Crisis and the Future of Gender Equality, London, Routledge, 17-36.

San José, Begoña (2014), «Igualdad versus austeridad: Resistencia, protestas y propuestas del movimiento feminista, Revista de Investigaciones Feministas 5, 185-206.

Squires, Judith (2007), The new politics of gender equality, New York, Palgrave Macmillan.

Stratigaki, Maria (2005), «Gender Mainstreaming vs Positive Action: An Ongoing Conflict in EU Gender Equality Policy», European Journal of Women's Studies 12(2), 165-186.

Valiente, Celia (2006), El Feminismo de Estado en España. El Instituto de la Mujer 1983-2003, Valencia, Universidad de Valencia.

Walby, Sylvia (2013), «Finance versus Democracy? Theorizing Finance in Society», Work, Employment and Society 27(3), 489-507.

Walby, Sylvia (2009), «Gender and the financial crisis», UNESCO Project on Gender and the Financial Crisis, [em linha] disponível em http://www.lancaster.ac.uk/fass/ 
doc library/sociology/Gender and financial crisis Sylvia Walby.pdf [consultado em 09/07/2015].

Alba Alonso. Investigadora postdoctoral de la Universidad de Santiago de Compostela. Ha participado en el proyecto europeo QUING, en diversos estudios impulsados por el Instituto Europeo para la Igualdad de Género y es miembro proyecto Gender Equality Policy in Practice. Es autora de El mainstreaming de género en España (Tirant lo Blanch, 2015) y co-editora de Ciencia Política con Perspectiva de Género (Akal, 2014).

E-mail: alba.alonso.alvarez@gmail.com

Departamento de Ciencia Política y de la Administración Campus Universitario Sur, s/n, 15782 - Santiago de Compostela, España.

Artigo recebido em 20 de maio de 2015 e aceite para publicação em 12 de julho de 2015. 